\title{
Practical Method to Derive Non-Linear Response Functions of Cameras for Scientific Imaging
}

\author{
HANAOKA, Yoichiro, SUZUKI, Isao, SAKURAI, Takashi \\ (NAOJ)
}

We developed a practical method to derive response functions which convert the amount of incident light to the A/D counts of cameras for scientific imaging [1]. Some cameras do show non-linear responses, and we have to know them to carry out photometric analysis with the data taken by such cameras. We applied this method for an InGaAs near-infrared camera, XEVACL-640 (XENICS), and succeeded to derive a response function, even though it was complicated non-linear one. In principle, such response functions can be derived from the output A/D counts, $S$, measured at various incident light levels, $I$, which are accurately controlled over the whole dynamic range. However, it is not easy to actually do such measurements with high accuracy. Then we developed a new practical method to derive non-linear response functions. In this method, we need a mechanism to accurately control the amount of incident light into cameras just within a limited dynamic range and at a limited number of steps (relative light level $T$, which may be exposure time, transparency, etc.). A variable brightness light source (brightness $B$ ), which supplies the incident light into cameras, is also necessary, but we do not need to know its accurate brightness.

Figure 1(a) shows the raw results measured under such conditions; output A/D values, $S$, are measured at several $T$ 's under various unknown $B$ 's (the brightness of the incident light, $I$, is written as $I=B T$ ). It seems that without knowing $B$ 's, it is difficult to derive the relation between $I$ and $S$ from the results shown in Figure 1(a). However, we can derive $I d S / d I$, because the relation $d I$ $=B d T$ based on $I=B T$ brings $d S / d T=B d S / d I$, and this means $T d S / d T=T B d S / d I=I d S / d I$. The value $T d S / d T$ can be derived from the known values, $T$ and $S$, and therefore, without knowing $B$, we can derive a unique relation between $T$ and $T d S / d T(=I d S / d I)$ as shown in Figure 1(b). The relation seen in Figure 1(b) can be converted to the relation between $I$ and $S$ through the numerical integration. Figure 1(c) shows the derived relation between $I$ and $S$, namely the response function of the camera. Obviously the function is non-linear. Figure 1(d) shows a close-up of the low-light level portion of Figure $1(\mathrm{c})$, and we can find a complex non-linear behavior.

We are using a XEVA camera for the polarimetry of the infrared solar light, which requires high accuracy measurements. Compensation of the non-linearity based on the derived function works well, and we have quantitatively high-quality observational data.
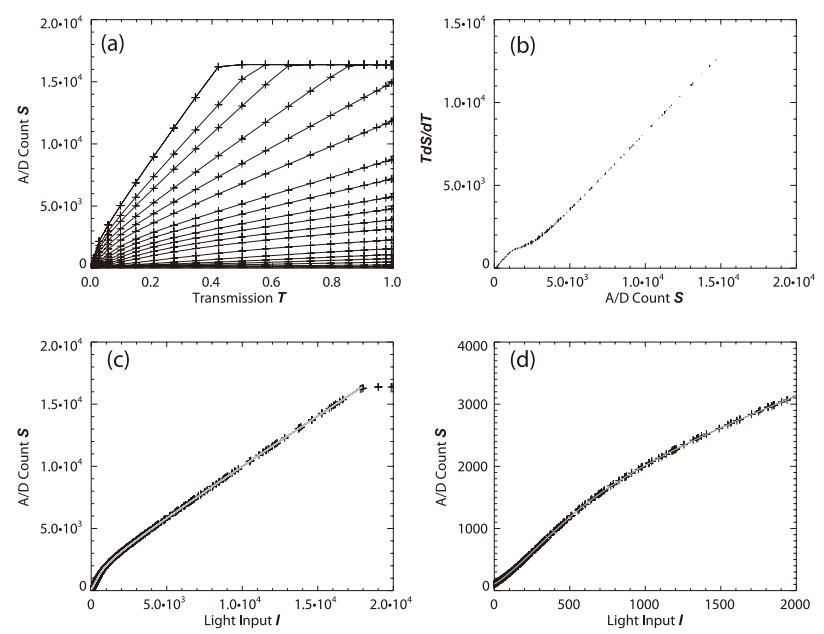

Figure 1: (a) Measured A/D counts $S$ at various values $T$ are shown with plus signs. Each line represents the measurements carried out under the same brightness $B$ of the light source. (b) Relation between the measured A/D count, $S$, and the $T d S / d T$ value. All the measured points are on a unique curve, regardless of the values of $B$. (c) Calculated non-linear response function between the amount of light input, $I$, and the output $\mathrm{A} / \mathrm{D}$ count, $S$, is shown with a solid grey line. The measured points shown in Figure 1(a) are also plotted with plus signs. (d) Enlargement of the low- $I$ range of panel (c).

\section{Reference}

[1] Hanaoka, Y., Suzuki, I., Sakurai, T.: 2011, App. Opt., 50, 2401-2407. 